\title{
ESTUDO COMPARATIVO ENTRE EXERCÍCIOS REALIZADOS NO SOLO E NA ÁGUA AQUECIDA DURANTE A FASE GESTACIONAL: ESTUDO DE CASO
}

\section{COMPARATIVE STUDY BETWEEN EXERCISES CONDUCTED ON THE GROUND AND IN HEATED WATER DURING PREGNANCY: A CASE STUDY}

\author{
Djenifer Queiroz de Souza ${ }^{1}$ \\ Izabela dos Santos Mendes ${ }^{1}$ \\ Karina da Silva Marson ${ }^{2}$ \\ Susane Moreira Machado ${ }^{3}$ \\ Ana Carolina Lacerda Borges ${ }^{4}$ \\ Paulo Roberto Garcia Lucareli ${ }^{5}$ \\ Mário Oliveira Lima ${ }^{6}$ \\ Fernanda Pupio Silva Lima ${ }^{6}$
}

RESUMO: O período gestacional é marcado por alterações musculoesqueléticas que podem resultar em dor e limitações funcionais. Este estudo teve como objetivo avaliar os efeitos terapêuticos de exercícios realizados sob imersão em água aquecida e no solo sobre o sistema musculoesquelético de uma gestante. Foi avaliada uma voluntária grávida de feto único, nulípara, com idade gestacional média de 32 semanas. A gestante foi submetida à avaliação do quadro álgico por meio da escala visual analógica, análise eletromiográfica da musculatura paravertebral e, à perimetria dos membros inferiores para quantificar o edema, antes e após aplicação do tratamento. Foram realizadas duas sessões de 50 minutos, sendo uma no solo e outra em piscina aquecida, com conduta composta de caminhada, alongamentos e relaxamento. Os resultados demonstraram que, após a realização do tratamento houve atenuação dos parâmetros avaliados, todavia, sendo mais satisfatório ao fim do protocolo hidroterapêutico. Concluiu-se que o programa de exercícios na piscina terapêutica proporcionou melhores resultados em relação ao solo no alívio da sintomatologia dolorosa e dos desconfortos da voluntária em questão.

Palavras-chave: gestação; sistema musculoesquelético; hidrocinesioterapia.

ABSTRACT: The gestational period is marked by musculoskeletal changes that can result in pain and functional limitations. This study evaluated the therapeutic effects of exercises done while immersed in warm water and on the floor on the musculoskeletal system of a pregnant woman. The volunteer was pregnant with a single fetus, nullipara, with an average gestational age of 32 weeks. She was evaluated for pain with the visual analog scale, electromyography of the paravertebral muscles, and the perimeter of lower limbs to quantify the edema, before and after treatment. Two, 50 minute sessions, one on the floor and another in a heated swimming pool, were conduct that consisted of walking, stretching, and relaxation. After treatment, there was attenuation of assessed parameters; however, with more satisfactory results at the end of the hydrotherapeutic protocol. Therefore, the exercise program in a therapeutic poll obtained better resulted in relation to the floor in relieving painful symptoms and discomforts in the volunteer in question.

Keywords: pregnancy; musculoskeletal system; aquatic therapy.

\footnotetext{
${ }_{1}^{1}$ Mestranda em Engenharia Biomédica - Univap. E-mails: djeniferqs@hotmail.com; izasmendes@hotmail.com.br.

${ }^{2}$ Graduada em Fisioterapia - Univap. E-mail: ka.marson@hotmail.com.

${ }^{3}$ Mestre em Engenharia Biomédica - Univap e docente da Univap. E-mail: susanetkd@hotmail.com.

${ }^{4}$ Graduanda em Fisioterapia - Universidade do Vale do Paraíba - Univap. E-mail: carolborges.fisio@gmail.com.

${ }^{5}$ Doutor em Ciências (Fisiopatologia Experimental) - Universidade de São Paulo - USP e docente da Universidade Nove de Julho. E-mails: paulolucareli@uninove.br; plucareli@hotmail.com.

${ }^{6}$ Doutor(a) em Engenharia Biomédica - Univap e docente da Univap. E-mails: mol@univap.br; fpupio@univap.br.
} 


\section{INTRODUÇÃO}

A gravidez é uma condição especial de saúde que traz diversas modificações no organismo materno, as quais são necessárias para o desenvolvimento fetal. Todavia, tais alterações podem afetar o funcionamento habitual dos sistemas digestório, circulatório, respiratório e musculoesquelético, refletindo na biomecânica corporal da gestante, podendo resultar em dor e limitações funcionais (BARACHO, 2002).

Segundo Rezende (1998) e Artal, Wiswel e Drinkwalter (2000), o sistema endócrino está intimamen-te relacionado às alterações musculoesque-léticas, durante a gravidez, devido ao aumento da excreção de hormônios, como a relaxina, o estrógeno e a progesterona, os quais promovem relaxamento ligamentar, amolecimento cartilaginoso e aumento no volume do líquido sinovial, gerando hipermobilidade e instabilidade articular, que, na pelve, é a causa da marcha anserina. No entanto, a partir do terceiro trimestre, nota-se redução da mobilidade articular devido à retenção hídrica, causando edema em tornozelos, parestesias, fraqueza muscular e dores nos punhos (KISNER; COLBY, 2004).

Outras alterações observadas incluem o crescimento das mamas em função do sistema de irrigação e aumento do fornecimento de sangue (REZENDE; MONTENEGRO, 1999), e do útero, que deixa de ser um órgão pélvico, tornando-se um órgão abdominal (KISNER, 2004).

Tais condições contribuem para a alteração do centro de gravidade materno, onde a pelve tende a rodar sobre o fêmur, acentuando a anteversão pélvica e a lordose lombar, a fim de evitar possíveis quedas (ARTAL; WISWEL; DRINKWALTER, 2000).
Como forma de compensar a hiperlordose lombar, pode ocorrer flexão da coluna cervical, deixando a cabeça mais anteriorizada, além de hiperextensão dos joelhos, alargamento da base de suporte e transferência de peso para os calcâneos (LOPES; ANDRADE, 1995; ARAUJO, 1997).

As principais alterações no sistema circulatório ocorrem no débito cardíaco e distribuição dos fluxos sanguíneos regionais. O débito cardíaco aumenta de 30 a $60 \%$ e a pressão sanguínea cai no primeiro trimestre, em decorrência da distensibilidade venosa (REZENDE, 1998). Ocorre, ainda, aumento do plasma maior que o de hemácias, podendo provocar uma anemia fisiológica (KISNER; COLBY, 2004).

Já, no sistema respiratório, as alterações são tanto anatômicas quanto funcionais e ocorrem antes mesmo que o crescimento uterino influencie na ventilação (ARTAL; WISWEL; DRINKWALTER, 2000; MONTENEGRO, 1999). Entre as modificações anatômicas, destacam-se o deslocamento do diafragma, $4 \mathrm{~cm}$ para cima, e o alargamento das últimas costelas, o que confere aumento nos diâmetros transverso do tórax e ântero-posterior em cerca de $2 \mathrm{~cm}$, além do ângulo subcostal que passa de $68^{\circ}$, no início da gestação, para $103^{\circ}$ ao fim desta (LOPES; ANDRADE, 1995; PARRISHI; CARLSON, 1998).

Segundo Rezende (1998), em relação às alterações funcionais, a capacidade vital não se altera significativamente, porém o volume minuto, o volume corrente e a frequência respiratória tendem aumentar progressivamente, o que é contradito por Kisner e Colby (2004) e Valadares (apud SOUZA, 1999), que afirmaram não haver alterações na frequência respiratória que se mantém constante durante todo o período 
gestacional.

Em virtude das várias adaptações sofridas pelo organismo materno, o tratamento fisioterapêutico visa à monitorização das alterações físicas, enfocando a manutenção do bem-eestar (KISNER; COLBY, 2004).

Desse modo, dispõe da cinesioterapia e hidroterapia. A cinesioterapia é um método terapêutico que utiliza o movimento para restabelecer a função. Essa técnica baseiase em exercícios de resistência à fadiga, fortalecimento, mobilidade e flexibilidade, relaxamento e coordenação motora (GUIMARÃES; CRUZ, 2003; KISNER; COLBY, 2004).

A hidroterapia emprega os princípios físicos da água, como o empuxo, a pressão hidrostática, a termocondutividade e o efeito diurético, em conjunto com a cinesioterapia. A água possui propriedades físicas peculiares que a transformam em um meio terapêutico eficiente e seguro para a realização de exercícios (KISNER; COLBY, 2004; THOMSON; SKINNER; PIERCY, 1994).

De acordo com Bates e Hanson (1998) exercícios realizados em imersão trazem inúmeras vantagens às gestantes, como relaxamento, redução do espasmo muscular e da sensibilidade à dor, além de facilitar a movimentação articular.

Tem-se o conhecimento de que a atividade física pode trazer grandes benefícios no tratamento dos desconfortos musculoesqueléticos apresentado pelas gestantes. No entanto, a literatura aponta que há poucos estudos comparativos entre exercícios realizados no solo e na água aquecida e seus efeitos terapêuticos na qualidade de vida.

\section{OBJETIVO}

Este estudo teve como objetivo avaliar os efeitos terapêuticos de exercícios realizados sob imersão em água aquecida e no solo sobre o sistema musculoesquelético de uma gestante.

\section{METODOLOGIA}

Trata-se de um estudo experimental transversal e comparativo entre exercícios realizados no solo e em piscina aquecida, na fase gestacional.

A voluntária foi acompanhada no Centro de Práticas Supervisionadas (CPS) da Faculdade de Ciências da Saúde (FCS) Universidade do Vale do Paraíba, após a assinatura do termo de consentimento livre e esclarecido e aprovação do projeto pelo Comitê de Ética e Pesquisa da Universidade sob o número do protocolo H130/CEP2010.

Participou desta pesquisa uma gestante, com ausência de doenças clínicas ou obstétricas e idade gestacional média de 32 semanas.

Como critérios de inclusão, foram considerados: indicação médica para fisioterapia e idade gestacional mínima de 26 semanas; foram excluídas as gestantes que apresentavam hipertensão arterial, placenta prévia, cérvix incompetente, ruptura de membranas, retardo no crescimento intra-uterino, dilatação do colo, sangramento vaginal, perda de líquido amniótico, parto prematuro prévio, doença cardíaca materna, doença cutânea infectocontagiosa, insuficiência renal e fobia de água.

A avaliação, para controle da melhora clínica, foi realizada no Laboratório de Biodinâmica do CPS, por meio dos seguintes métodos: 
- Eletromiografia (EMG), para analisar a atividade muscular dos paravertebrais. $O$ potencial mioelétrico foi captado por meio de um eletromiógrafo de superfície, 4 canais $\left(\right.$ EMG System $\left.{ }^{\circledR}\right)$, acoplado à bateria, a fim de evitar interferência da rede elétrica, com frequência de amostragem de $2.000 \mathrm{~Hz}$. Os eletrodos foram fixados a dois centímetros laterais do processo espinhoso da $1^{\text {a }}$ vértebra lombar (L1), conforme o protocolo Surface-EMG for the Non Invasive Assessment of Muscle (SENIAM). Para atenuar a impedância tecidual, foi realizada higienização com algodão embebido de álcool, a $70 \%$, no local de fixação dos eletrodos. Os dados foram coletados em posição ortostática, durante 15 segundos, a partir de três situações distintas, sendo estas, repouso $(R)$, atividade $(A-$ partindo da flexão máxima do tronco em direção à extensão máxima) e flexo-extensão ( $F$ flexões e extensões do tronco repetidas). Concomitantemente à coleta, o sinal da atividade elétrica paravertebral foi submetido à filtragem disponibilizada pelo aparelho. $\mathrm{O}$ processamento dos sinais foi realizado por meio do software EMGWork Analysis, onde os dados obtidos foram plotados, para que fosse possível adquirir os valores de RMS. As contrações foram selecionadas visualmente, desconsiderando os primeiros 5 segundos de coleta;

- Perimetria dos membros inferiores, realizada em ortostatismo, para quantificar o edema gravitacional. Para tal, foram prédeterminados pontos, a partir de referências anatômicas, os quais foram demarcados na pele da voluntária, com lápis dermográfico e quantificados por meio de fita métrica, adotando-se a unidade de medida em centímetros;

- Escala Visual Analógica (EVA), para avaliar a presença e/ou intensidade da dor. A gestante foi orientada a comparar a dor apresentada com a maior dor já sentida, cujo valor era correspondente a 10 na escala.

Durante as sessões, foram monitorados os sinais vitais, como a frequência cardíaca, a pressão arterial e a frequência respiratória, para controle da intensidade dos exercícios.

Seguida da avaliação, a voluntária foi submetida a duas sessões de tratamento, uma em piscina aquecida e outra no solo, com intervalo de quinze dias entre eles, constituindo, respectivamente, o protocolo hidroterapia e o protocolo solo.

As sessões dos exercícios terapêuticos tiveram duração de 50 minutos e compreenderam três fases: aquecimento, alongamento e relaxamento.

O primeiro atendimento foi realizado no Setor de Hidroterapia com piscina coberta e aquecida, à temperatura de $34^{\circ} \mathrm{C}$, com o nível de água para a realização dos exercícios na altura do processo xifóide. 0 segundo atendimento foi realizado no Setor de Cinesioterapia da FCS.

Foi realizado o mesmo protocolo de tratamento na piscina e no solo, com o mesmo número de séries, repetições e tempo, exceto os exercícios de relaxamento.

Inicialmente, foi realizada uma caminhada leve, para frente, para trás e com passada lateral, durante 15 minutos, para aquecimento; seguido de alongamento (2 séries de 20 segundos), para a região cervical, membros superiores, tronco e membros inferiores; tração lombar (3 séries de 1 minuto); relaxamento final, durante 15 minutos, em que foram realizados movimentos de serpenteio, na água e no 
solo, exercícios respiratórios com a gestante posicionada em decúbito dorsal, adotando o padrão $1: 1$, ou seja, inspiração nasal profunda diafragmática, seguida de expiração oral freno labial, com pausas para o descanso e mudança de decúbito a cada 8 movimentos respiratórios, evitando a compressão da veia cava inferior e consequente hipotensão.

A voluntária foi orientada a não realizar exercícios com a bexiga cheia, não realizar manobras de valsalva e se hidratar com água durante os atendimentos.

\section{RESULTADOS}

Posteriormente à análise dos dados coletados, observa-se, na voluntária, após a realização do tratamento hidroterapêutico, atenuação do potencial mioelétrico em $\mathrm{A}$ (42,2 para 42), contudo nota-se aumento da atividade muscular paravertebral em $\mathrm{R}(10,8$ para 11,8$)$ e $F(32$ para 38,3$)$.

Os resultados obtidos, após o tratamento no solo, demonstram, na paciente avaliada, uma diminuição significativa da atividade paravertebral em $A$ $(40,8$ para 30,2$)$ e $F(54,3$ para 44,0$)$, enquanto em $R$ observa-se aumento do potencial mioelétrico $(35,6$ para 47,5$)$.

Quando comparados os resultados eletromiográficos obtidos após a realização dos protocolos de tratamento, a Fig. 1 revela atenuação do potencial mioelétrico predominante, no protocolo solo, havendo redução de $26 \%$ em $A$ e 18,6\% em F, porém um aumento de $30 \%$ em R. Nota-se, no protocolo hidroterapêutico, diminuição da atividade elétrica paravertebral apenas em A $(0,5 \%)$, sendo apresentado aumento de 9,2 e $20,7 \%$, respectivamente, em $\mathrm{R}$ e F.

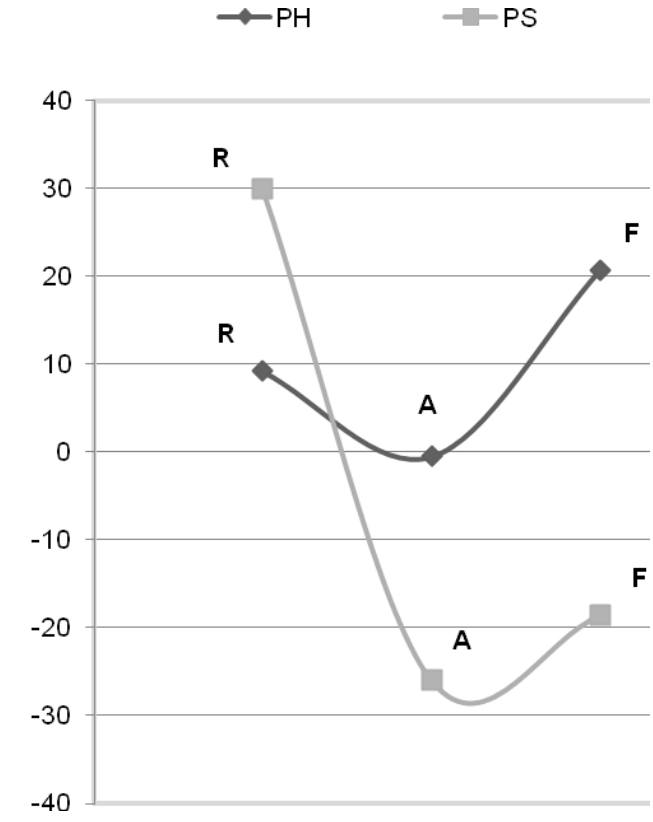

Fig. 1 - Distribuição porcentual (\%) comparativa da atividade eletromiográfica após o protocolo solo (PS) e protocolo hidroterapia (PH).

Os resultados obtidos na EVA, que avaliou a intensidade da dor, mostraram redução dos valores, apresentados anteriormente pela voluntária, conforme indicado na Tabela 1. Tal redução foi predominante após - protocolo hidroterapia $(\mathrm{PH})$ com diminuição de $75 \%$, enquanto o protocolo solo (PS) apresentou diminuição de $37,5 \%$.

Tabela 1 - Distribuição porcentual (\%) da intensidade de dor lombar antes e após tratamento

\begin{tabular}{lcccc}
\hline & \multicolumn{2}{c}{ PH } & \multicolumn{2}{c}{ PS } \\
& M & DP & M & DP \\
\hline Antes & 35,4 & $\pm 12,52$ & 35,6 & $\pm 12,35$ \\
Após & 34,8 & $\pm 12,48$ & 35,25 & $\pm 12,22$ \\
\hline
\end{tabular}

O edema gravitacional apresentou diminuição dos valores iniciais após a realização de ambos os protocolos, todavia, com resultados mais satisfatórios após o protocolo hidroterapêutico, em que observa- 
se, na gestante, redução média de 3,03\% após o PH e 1,94\% após o PS, conforme representado na Tabela 2.

Tabela 2 - Distribuição média (M) e desvio padrão (DP) da perimetria dos membros inferiores antes e após ambos os tratamentos

\begin{tabular}{ccc}
\hline & PH & PS \\
\hline Antes & 8 & 8 \\
Após & 2 & 5 \\
$\%$ & -75 & $-37,5$ \\
\hline
\end{tabular}

\section{DISCUSSÃO}

A gravidez é um período marcado por alterações fisiológicas que interferem na biomecânica corporal da gestante, podendo gerar desconfortos.

De acordo com Martins e Silva (2002), dentre as diversas adaptações sofridas pelo organismo materno, as que mais acarretam desconfortos são as musculoesqueléticas, entre as quais destaca-se a lombalgia.

Martins e Silva (2002), ao estudarem a prevalência de dores nas costas na gestação, identificaram que, aproximadamente, $80 \%$ das gestantes apresentam algia na coluna vertebral, em algum período gestacional, com predominância lombar e sacro ilíaca. Tais achados foram equivalentes aos verificados nos estudos de Östgaard (1996), Carlson et al. (2003) e Stuge, Hilde e Vollestad (2003), em que, aproximadamente, $50 \%$ das gestantes apresentaram algia lombar, e evidenciado no atual estudo.

Para Sant'ana et al. (2006), a dor lombar pode ser considerada de etiologia multifatorial, em que as principais causas são as hormonais, devido ao aumento da secreção de relaxina que leva à frouxidão ligamentar e, consequentemente, às dores; vasculares, em decorrência da diminuição do fluxo sanguíneo, gerando dor, principalmente, no terceiro trimestre da gestação e, por fim, biomecânicas, pelas adaptações fisiológicas que podem resultar em compressão das raízes nervosas da coluna lombossacra e tensão dos músculos espinhais antigravitacionais.

Há consenso, entre diversos autores, com relação à indicação do exercício físico como forma alternativa de tratamento para a lombalgia gestacional. De acordo com Colliton (1996), Hartmann e Bung (1999), Artal, Wiswel e Drinkwalter (2000) e Kisner e Colby (2004), um programa de exercícios aplicados no perío-do gestacional pode prevenir a instalação do quadro álgico, possivelmente por permitir adaptações biomecânicas mais eficientes.

Foi evidenciado, neste estudo, que a intervenção fisioterapêutica, baseada num protocolo hidrocinesioterapêutico, proporcionou uma atenuação da sintomatologia álgica lombar em comparação com o protocolo aplicado no solo. Tal afirmação pode ser justificada pelos efeitos fisiológicos da água, destacando-se o empuxo, que promove maior sustentação do peso corpóreo, reduzindo 0 estresse articular; a temperatura, que gera uma vasodilatação, aquecendo a musculatura e favorecendo a redução do tônus muscular; a turbulência e a pressão hidrostática que estimulam as terminações nervosas cutâneas, provocando um extravasamento sensorial que aumenta - limiar da dor e, assim, há diminuição na percepção álgica. A imersão tem efeito ainda sobre o sistema nervoso simpático, deprimindo a produção de noradrenalina e proporcionando uma sensação de bem-estar (SKINNER; THOMSON, 1985; BECKER; ANDREW, 2000). 
Neste estudo, foi estipulado o intervalo de 15 dias entre a aplicação dos dois protocolos de tratamento para evitar influência de um recurso terapêutico sob o outro nos resultados. Acredita-se que apenas um atendimento de hidroterapia não tenha promovido interferência na biomecânica da gestante, de maneira que pudesse promover o alívio da dor permanente até a aplicação dos exercícios no solo. Considerase, também, que, após o atendimento, a gestante retornou para suas atividades cotidianas normais, e a tendência é que haja uma acentuação do desconforto a cada dia, devido ao desenvolvimento do feto.

Estudos realizados por Sihvonen et al. (1998) constataram que quanto menor a atividade paravertebral, no início da gestação, maior a predisposição do desenvolvimento de dor e incapacidades no transcorrer desta.

Segundo Gonzaga (1999) e Martins e Silva (2002), a queixa álgica lombar, observada nas gestantes, é provocada pela grande pressão imposta às vértebras lombares mais baixas, predispondo os discos ao deslizamento. Tal condição devese à hipotonia dos músculos abdominais, com consequente fadiga dos músculos dorsais, potencialmente exigidos devido às adaptações necessárias ao período.

Observou-se, no atual estudo, discreto relaxamento da musculatura posterior do tronco, evidenciado pela atenuação da atividade paravertebral, após aplicação de ambos os protocolos de tratamento, todavia, com resultados mais satisfatórios ao fim do protocolo solo, a despeito dos efeitos fisiológicos decorrentes da imersão em água aquecida.

De acordo com Bates e Hanson (1998) e Koury (2002), a temperatura e o empuxo, em conjunto, reduzem o espasmo por favorecerem 0 aumento da circulação periférica. Para Baracho (2002), a água, além de exercer um efeito relaxante sobre a musculatura, diminuindo a tensão, permite maior sustentação do peso corporal, diminuindo o impacto dos exercícios sobre as articulações.

Assim, o achado deste estudo pode ser justificado pelo fato da gestante, após deixar o meio aquático, sentir novamente o sobrepeso advindo da gravidez, antes atenuado pelo empuxo. Essa condição pode contribuir para uma hiperextensão do tronco na tentativa de evitar possíveis quedas. Sugere-se, em novos estudos, a utilização de eletrodos subaquáticos, a fim de minimizar tal efeito.

Outra importante queixa das gestantes é o edema evidenciado principalmente nos membros inferiores. $\mathrm{O}$ aumento da secreção hormonal favorece a retenção hídrica, podendo levar à diminuição da mobilidade dos punhos e tornozelos, principalmente no terceiro trimestre gestacional, além de promover relaxamento ligamentar, contribuindo com o maior risco de contusões, o que justifica a importância do alongamento muscular num programa de exercícios (ARTAL; WISWEL; DRINKWALTER, 2000; VERDERI, 2006).

Para Hartmann e Bung (1999), a prática de atividade física regular, durante a gestação, pode minimizar a ocorrência de dores nas mãos e membros inferiores, possivelmente, por promover menor retenção de líquido no tecido conectivo.

Estudos realizados por Horns, Ratcliffe e Leggett (1996) compararam primíparas sedentárias e praticantes de atividade física regular, durante 0 terceiro trimestre gestacional, e constataram que a prática de 
atividade física estava relacionada com a redução do edema observado nas voluntárias.

No atual estudo, observou-se a diminuição do edema gravitacional predominantemente após a realização do protocolo hidroterapêutico, visto que a imersão, em decorrência dos efeitos do empuxo e da pressão hidrostática, favorece - controle do edema gravídico pela facilitação do retorno venoso e linfático, com consequente incremento da diurese (PREVEDEL et al., 2003).

Como fator limitante deste estudo, foi considerado o pequeno número da amostra, atribuída a dificuldade na obtenção de liberação médica, devido à contra-indicação, para a realização de exercícios, além dos desconfortos apresentados por elas, como consequências da própria gestação.

\section{CONCLUSÃO}

O programa hidroterapêutico estabelecido, neste estudo, proporcionou melhores resultados em relação ao solo na diminuição do edema gravitacional dos membros inferiores, na tensão dos músculos dorsais e, consequentemente, da intensidade das dores lombares na paciente em questão.

\section{REFERÊNCIAS}

ARAÚJO, D. Expecting Questions about Exercise and Pregnancy. Phys. sport med., v. 25, n. 4, p. 85-93, 1997.

ARTAL, R.; WISWEL, R. A.; DRINKWALTER, B. L. O exercício na gravidez. 2. ed. São Paulo: Manole, 2000.

BARACHO, E. Fisioterapia aplicada à obstetrícia: aspectos de ginecologia e neonatologia. Rio de Janeiro: Médica e Científica, 2002.

BATES, A.; HANSON, N. Exercícios Aquáticos Terapêuticos. 1. ed. São Paulo: Manole, 1998.

BECKER, B. E.; ANDREW, J. C. Terapia Aquática Moderna. São Paulo: Manole, 2000.

CARLSON, H. L. et. al. Understanding and managing the back pain of pregnancy. Curr Womens Health Rep., v. 3, p. 65-71, 2003.

COLLITON, J. Back pain and pregnancy: Active management strategies. Phys. Sportsmed., v. 24, p. 1-6, 1996,.

GONZAGA, C. S. Considerações gerais sobre a prática de ginástica para gestante. 1999. Monografia (Aperfeiçoamento /Especialização em Treinamento Desportivo) - Universidade Estadual de Maringá Maringa, 1999.

GUIMARÃES, L. S.; CRUZ, M. C. Exercícios terapêuticos: a cinesioterapia como importante recurso da fisioterapia. Lato\&Sensu, v. 4, n. 1, p. 3-5, 2003.

HARTMANN, S.; BUNG, P. Physical exercise during pregnancy; physiological considerations and recommendations. J. Perinat. Med., v. 27, p. 204-215, 1999.

HORNS, P. N.; RATCLIFFE, L. P.; LEGGETT, J.C. Pregnancy outcomes among active and sedentary primiparous women. J. obstet. gynecol. neonatal nurs., v. 25, p. 4954, 1996.

KISNER, C.; COLBY, L. A. Exercícios Terapêuticos: Fundamentos e Técnicas. 4 ed. São Paulo: Manole, 2004.

KOURY, J. Programa de Fisioterapia Aquática: Um Guia para Reabilitação ortopédica. São Paulo: Manole, 2002. 
LOPES, C. M.; ANDRADE, J. Programação de exercícios na gravidez. Rev. bras. med. ginecol. obstet., v. 6, n. 4, p. 222-224, 1995.

MARTINS, R. F.; SILVA, J. L. P. Algias posturais na gestação: prevalência e tratamento. 2002. 122f. Dissertação (Mestrado em Tocoginecologia) - Faculdade de Ciências Médicas, Universidade Estadual de Campinas, 2002.

MONTENEGRO, C. A. B. Obstetrícia Fundamental. 8. ed. Rio de Janeiro: Guanabara Koogan, 1999.

ÖSTGAARD, H. C. Assessment and treatment of low back pain in working pregnant women. Spine, v. 16, n. 1, p. 61-69, 1996.

CARLSON, B.; PARRISH, D. Exercisimg during pregnancy: What to tell yuor patients. Women's health prim. care, v. 1, n. 2, p.171179, mar. 1998.

PREVEDEL, T. T. S. et al. Repercussões maternas e perinatais da hidroterapia na gravidez. Rev. bras. ginecol. obstet., v. 25, n. 1, p. 53-59, 2003.

REZENDE, J. Obstetrícia. 8. ed. Rio de Janeiro: Guanabara Koogan, 1998, p. 104150.

SANT'ANA, P. F. et al. Caracterização da dor lombar em gestantes atendidas no hospital Universitário de Brasília. Universitas: Ciências da saúde, v. 4, n. 1-2, p. 37-48, 2006.

SIHVONEN, T. et al. Functional changes in back muscle activity correlate with pain intensity and prediction of low back pain during pregnancy. Arch. phys. med. reabil., v. 79, p. 1210-2, 1998.

SKINNER, A. T.; THOMSON, A. M. Duffield Exercícios na água. 3 ed. São Paulo: Manole, 1985.

STUGE, B.; HILDE, G.; VOLLESTAD, N. Physical therapy for pregnancy - related low back and pelvic pain: a systematic review. Acta obstet. gynecol. scand., v. 82, p. 983990, 2003.

THOMSON, A.; SKINNER, A.; PIERCY, J. Fisioterapia de Tidy. São Paulo: Santos, 1994.

VALADARES, J. D. Interações Fisiológicas na Gravidez. In: SOUZA, E. L. B. L. Fisioterapia aplicada à obstetrícia e aspectos de neonatologia. 2. ed. rev. e amp. Belo Horizonte: Helth, 1999, p. 41-56.

VERDERI, E. Gestante: Elaboração de programas de exercícios. São Paulo: Porte, 2006, p. 19-32. 